\title{
Lumbar Hernia: An Unusual Presentation of Bear Maul
}

\author{
Mubashir Ahmad Shah ${ }^{1}$, Aakib Hamid Charag ${ }^{1 *}$, Adil Pervaiz Shah ${ }^{1}$, Haroon Rashid Zargar ${ }^{2}$ \\ ${ }^{1}$ Department of General \& Minimal Access Surgery, Sher-I-Kashmir Institute of Medical Sciences, Srinagar, India; ${ }^{2}$ Department of \\ Plastic \& Reconstructive Surgery, Sher-I-Kashmir Institute of Medical Sciences, Srinagar, India. \\ Email: drmashah2@yahoo.co.in, *ahc.gmc@gmail.com,dradilpervaiz@gmail.com,hrzargar@yahoo.co.in
}

Received July $1^{\text {st }}, 2013$; revised August $5^{\text {th }}, 2013$; accepted September $1^{\text {st }}, 2013$

Copyright (C) 2013 Mubashir Ahmad Shah et al. This is an open access article distributed under the Creative Commons Attribution License, which permits unrestricted use, distribution, and reproduction in any medium, provided the original work is properly cited.

\begin{abstract}
Introduction: Typical lumbar hernias are very rare surgical conditions. Lumbar hernias can be congenital or acquired. About $25 \%$ of all lumbar hernias have a traumatic etiology. Case-Report: We here reported a case of a 55-year-old male who was mauled by a bear. The patient developed an atypical lumbar hernia after 6 months of sustaining craniofacial and abdominal trauma. Open hernioplasty, which was a very challenging job, was done in this patient. Conclusion: Post traumatic lumbar hernias have been reported but till today no case of lumbar hernia following an attack by a bear has been reported in literature. Both acute and long-standing post-traumatic lumbar hernias are rare but challenging conditions. The corrective surgical procedure becomes more complex as hernial defect enlarges. Reconstruction is a challenging aspect of lumbar hernia surgery.
\end{abstract}

Keywords: Lumbar; Hernia; Trauma; Bear Maul

\section{Introduction}

Typical lumbar hernias are very rare surgical conditions. Lumbar hernias can be congenital or acquired. About $25 \%$ of all lumbar hernias have a traumatic etiology [1].

The trauma may be post surgical due to the blunt and penetrating trauma to the flank. We report the case of a male patient who presents with a lumbar hernia, 6 months following a bear maul.

\section{Case Report}

A 55-year-old male patient presented with history of being attacked by bear about 6 months back with trauma face and abdomen. Apart from the facial trauma, the patient had a healed lumbar laceration. Facial trauma was managed by the department of plastic surgery. Lumbar laceration was closed primarily at the time of presentation after ruling out intra-abdominal injury. After six months on follow up of plastic and reconstructive surgery patient presented with a swelling in left lumbar region associated with a dragging pain. Patient was referred to the department of general surgery for the management of same.

On examination, there was a $20 \times 15 \mathrm{~cm}$ non-tender, non-pulsatile and reducible swelling in the left lumbar region with an expansile cough impulse, with palpable gut loops within it. There was about $10 \times 8 \mathrm{~cm}$ palpable defect under the swelling getting more prominent after reducing the hernia. There was a scar of old repaired laceration visible on the swelling. The examination of rest of the abdomen, right flank, back was normal. Plain radiograph of the abdomen revealed gas shadows (gut loops) within the swelling (Figure 1). Ultrasonography revealed a $15 \times 13 \mathrm{~cm}$ ill-defined mass in the left lumbar region with areas of mixed echogenicity within the swelling. Computed tomography (CT) scan of the abdomen was carried out which confirmed the presence of left lumbar hernia containing gut loops part of transverse colon and small gut with a bony defect in left ilium (Figure 2). A diagnosis of a reducible post traumatic (bear maul) left lumbar hernia was made and patient was taken up for surgery. The patient was placed in a right lateral position. The swelling was explored through a left flank incision. The contents of the hernia were reduced back into peritoneal cavity. Fractured ilium and loss of attachment of abdominal muscles from the iliac crest was noted (Figure 3). Defect in the peritoneum was closed using absorbable suture. A prolene mesh was fashioned as inlay prosthesis and was placed in the extra peritoneal space through the defect in the muscle layer (Figure 4). The mesh was secured with prolene sutures and rest of the wound was closed in layers. The immediate postoperative period was uneventful. Oral feeds were started on the second postoperative day. The patient was discharged on 


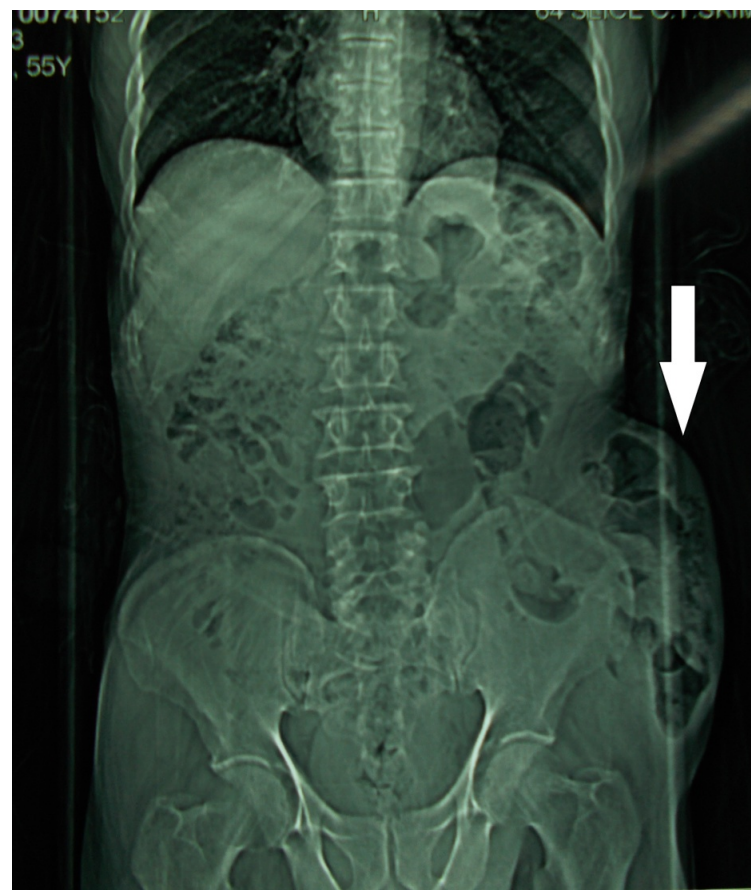

Figure 1. Erect abdominal radiograph showing herniated bowel loops in left lumbar region.

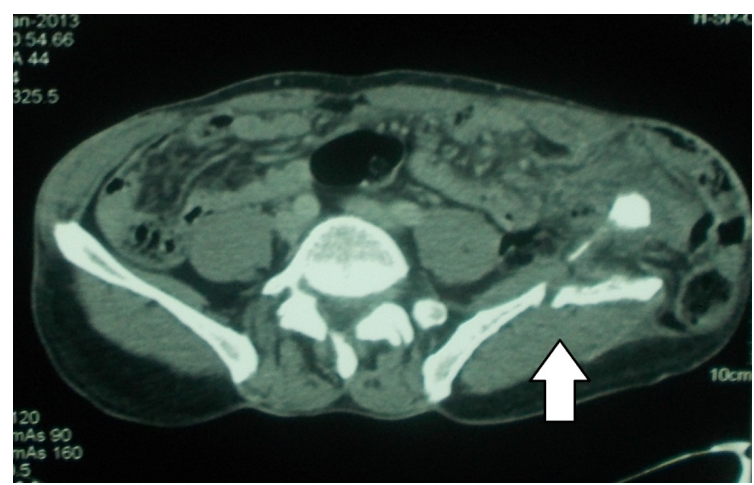

Figure 2. CT scan showing broken left ilium with bowel loops lateral to it.

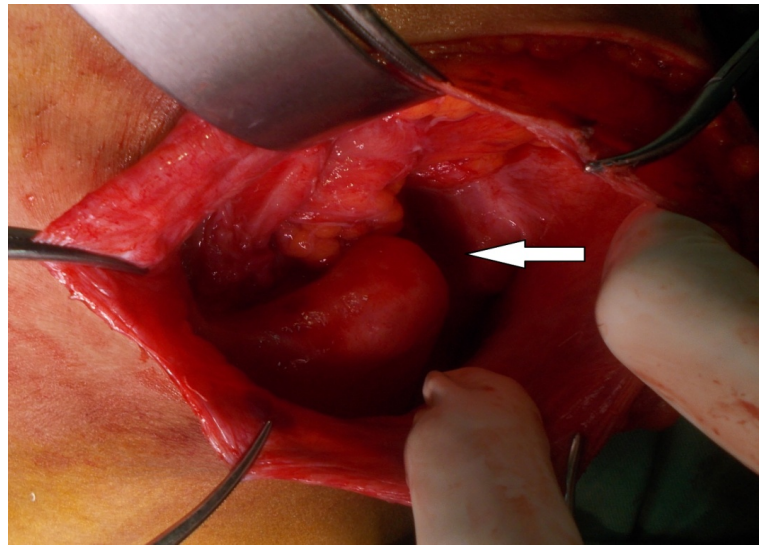

Figure 3. Intra operative picture showing broken ilium with loss of muscle attachments.

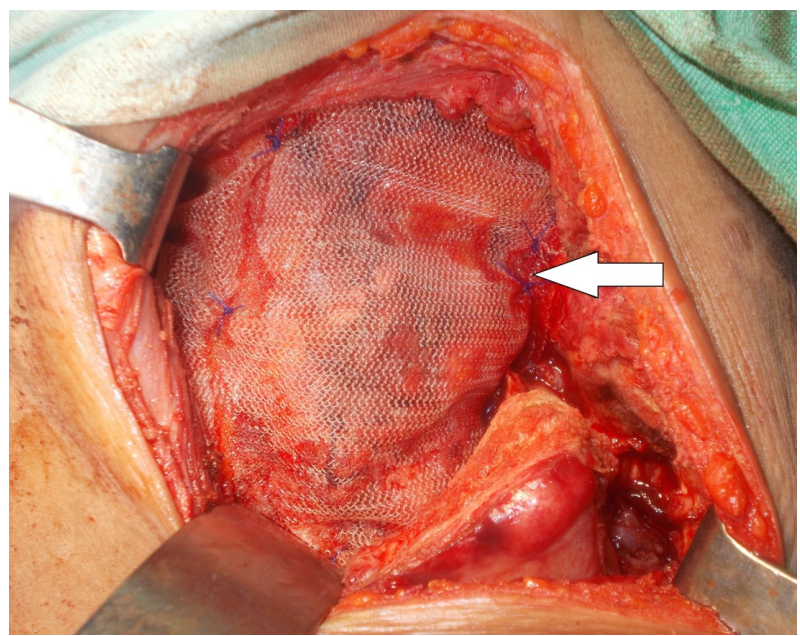

Figure 4. Placement of prolene mesh.

third post operative day. During the follow-up visit after one month, and three months the operation scar was found to be well healed and the patient was absolutely asymptomatic, with no recurrence.

\section{Discussion}

Lumbar hernias are relatively rare. These occur more commonly in males and are twice as common on the left, than on the right side. Patients are usually between 50 to 70 years old. These hernias can occur anywhere within the lumbar region but are more common through the superior lumbar triangle (of Grynfeltt-Lesshaft), an inverted triangle bounded by 12th rib, erector spinae and the posterior border of the internal oblique muscle. The inferior lumbar triangle (of Petit), bounded by iliac crest, posterior border of external oblique and the anterior border of latissimus dorsi is the next most frequent site [2]. Lumbar hernias have been classified as congenital (20\%) or acquired (80\%). If acquired, they may be primary (55\%) or secondary following trauma, surgery or inflammation (25\%). These hernias have a natural history of a gradual increase in size over time and may assume large proportions [3].

The differential considerations at this stage include lipoma, soft tissue tumors, hematoma or abscess. The hernia may contain retroperitoneal fat, kidney, colon or less commonly small bowel, omentum, ovary, spleen or appendix. On auscultation, bowel sounds may be audible over the swelling if the hernia contains bowel loops. Bowel incarceration occurs in $25 \%$ but strangulation is rare because of wide hernial neck [4]. Lateral or oblique radiograph of the lumbar region may show gas filled loops of the bowel lying outside the abdominal cavity. Upper or lower gastrointestinal contrast studies are useful in delineating the herniated bowel segment. In addition, an intravenous urogram may be performed to visualize any displacement of the kidney or ureter into the hernia. 
Ultrasonography may fail to demonstrate the hernia due to low index of suspicion and presence of fat. CT scan can accurately distinguish the muscular and fascial layers, detect the presence of a defect in these layers, visualize herniated fat or viscera and differentiate a hernia from a hematoma, abscess or soft-tissue tumor $[5,6]$. The goal of hernia repair is to eliminate the defect and to construct an elastic and firm abdominal wall that will withstand the stress of daily physical activities. A lumbar hernia should be repaired surgically, as it is prone to both obstruction and strangulation $[7,8]$.

Techniques for repair include anatomical closure, overlapping of the aponeurosis, use of musculofascial flaps, prosthetic meshes and laparoscopic mesh repair in case of uncomplicated lumbar hernias [4]. Currently, extraperitoneal mesh repair is considered the optimal treatment for isolated unilateral lumbar hernia. The Rives Stoppa approach, wherein, a large rectangular mesh is fashioned to be placed in the pre-peritoneal space extending from umbilicus to retropubic space and between the two anterior superior iliac spines, appear to be the most promising open technique for bilateral lumbar hernia, recurrent hernias or multiple site hernias with comparatively low recurrence rates [8]. Extra- peritoneal position of the mesh is advantageous as no bony anchorage is essential. The weight of the intraperitoneal contents is an additional support to maintain the mesh in correct position in the early postoperative period. Laparoscopic transabdominal preperitoneal mesh repair for lumbar hernia confers all the benefits of minimal access surgery to the patient. It is a tensionless repair. It follows the current principle of hernia surgery and is based on the sound physiological principle of diffusing the total intraabdominal pressure on each square inch of the mesh implanted $[9,10]$.

\section{Review of Literature}

Post traumatic lumbar hernias have been reported but till date no case of lumbar hernia following an attack by a bear has been reported. Agarwal N and his colleagues [11] have reported a case of traumatic abdominal wall hernia in a 40-year-old female from North India who was hit by a bull. Al Sarela et al. [12] have reported a case of lumbar hernia which had developed following blunt abdominopelvic trauma. Burick et al. [13] reported a case of acute lumbar hernia as a direct result of blunt trauma which was explored and repaired laparoscopically. Moreno-Egea et al. [14] studied open versus laparoscopic lumbar hernia repairs. They concluded that outcomes did not differ with respect to morbidity and recurrence rate after long-term follow-up and that laparoscopic approach for lumbar hernia is safe, effective, and more efficient than open repair and can be considered the procedure of choice. Links and Berney [15] report the use of bone suture anchors placed in the iliac crest during transperitoneal laparoscopy for mesh fixation to repair a recurrent traumatic lumbar hernia.

\section{Conclusion}

Both acute and long-standing post-traumatic lumbar hernias are rare but challenging conditions, which require an appropriate index of suspicion and investigations for diagnosis and a well planned surgical repair. These hernias increase in size and become symptomatic. The corrective surgical procedure becomes more complex as hernial defect enlarges. Reconstruction is a challenging aspect of lumbar hernia surgery.

\section{REFERENCES}

[1] W. T. Swartz, “Lumbar Hernia,” In: L. M. Nyhus and R. E. Condon, Eds., Hernia, 2nd Edition, Lippincott, Philadelphia, 1978, pp. 409-426.

[2] J. E Skandilakis and J. B. Flament, "The Surgical Clinics of North America,” Vol. 80, 2000, pp. 388-391.

[3] W. P. Geis and G. T. Hodakowski, "Lumbar Hernia,” In: L. Nyhus and R. Condon, Eds., Hernia, 5th Edition, Lippincott, Philadelphia, 2001, pp. 425-427.

[4] B. Devlin and A. N. Kingsnorth, "Management of Abdominal Hernias,” 2nd Edition, Edward Arnold, London, 1998, pp. 330-334.

[5] M. E. Baker, J. L. Weinerth, R. T. Andriani, et al., "Lumbar Hernia: Diagnosis by CT,” American Journal of Roentgenology, Vol. 148, No. 3, 1987, pp. 565-567. http://dx.doi.org/10.2214/ajr.148.3.565

[6] K. L. Killeen, S. Girard, J. H. Demeo, et al., "Use of CT to Diagnose Traumatic Lumbar Hernia," American Journal of Roentgenology, Vol. 174, No. 5, 2000, pp. 14131415. http://dx.doi.org/10.2214/ajr.174.5.1741413

[7] G. E. Leber, J. L. Garb, A. l. Albert and W. P. Reed, "LongTerm Complications Associated with Prosthetic Repair of Incisional Hernias,” Archives of Surgery, Vol. 132, 1998, pp. 1141-1144.

[8] A. K. Meinke, "Totally Extraperitoneal Laparoendoscopic Repair of Lumbar Hernia,” Surgical Endoscopy and Other Interventional Techniques, Vol. 17, No. 5, 2003, pp. 734737. http://dx.doi.org/10.1007/s00464-002-8557-8

[9] B. T. Heniford, D. A. Iannitti and M. Gagner, "Laparoscopic Inferior and Superior Lumbar Hernia Repair,” Archives of Surgery, Vol. 132, No. 10, 1997, pp. 1141-1144. http://dx.doi.org/10.1001/archsurg.1997.01430340095017

[10] P. K. Chowbey, A. Sharma, R. Khullar, et al., "Laparoscopic Ventral Hernia Repair,” Journal of Laparoendoscopic \& Advanced Surgical Techniques, Vol. 10, No. 2, 2000, pp. 79-84. http://dx.doi.org/10.1089/lap.2000.10.79

[11] N. Agarwal, S. Kumar, M. K. Joshi and M. S. Sharma, "Traumatic Abdominal Wall Hernia in Two Adults: A Case Series,” Journal of Medical Case Reports, Vol. 3, 2009, p. 7324.

http://dx.doi.org/10.4076/1752-1947-3-7324 
[12] A. I. Sarela, A. A. Mavanur, A. Bhaskar, Z. F. Soonawala, G. Devnani, H. K. Shah, et al., "Post Traumatic Lumbar Hernia,” Journal of Postgraduate Medicine, Vol. 42, No. 3, 1996, pp. 78-80.

[13] A. J. Burick and S. A. Parascandola, "Laparoscopic Repair of a Traumatic Lumbar Hernia: A Case Report,” Journal of Laparoendoscopic Surgery, Vol. 6, No. 4, 1996, pp. 259-262. http://dx.doi.org/10.1089/lps.1996.6.259

[14] A. Moreno-Egea, A. C. Alcaraz and M. C. Cuervo, "Sur- gical Options in Lumbar Hernia: Laparoscopic versus Open Repair; A Long-Term Prospective Study,” Surgical Innovation, Vol. 20, No. 4, 2013, pp. 331-344. http://dx.doi.org/10.1177/1553350612458726

[15] D. J. Links and C. R. Berney, “Traumatic Lumbar Hernia Repair: A Laparoscopic Technique for Mesh Fixation with an Iliac Crest Suture Anchor,” Hernia, Vol. 15, No. 6, 2011, pp. 691-693.

http://dx.doi.org/10.1007/s10029-010-0716-6 